\title{
Integriertes Hochwassermanagement
}

2002 trat in Österreich an einigen Flüssen das größte bisher beobachtete Hochwasser auf. Mit neun Todesopfern und über 3 Mrd. € Schaden überstieg dieses Ereignis alle anderen seit 1965/66. In Kooperation mit dem Lebensministerium, dem BMVIT und den Bundesländern erstellte das Zentrum für Naturgefahren und Risikomanagement der Universität für Bodenkultur Wien im Rahmen der Plattform Hochwasser die erste umfassende, nationale Hochwasserdokumentation. Anschließend wurde das Projekt FloodRisk I (Analyse der Hochwasserereignisse vom August 2002 - FloodRisk) durchgeführt. Diese Analyse stellte den nächsten Schritt dar, um in Zukunft Grundlagen für strategische Entscheidungen im Sinne eines integrierten Hochwassermanagements zu besitzen. Das Hochwasser 2002 wurde in insgesamt 46 Teilprojekten aus den Bereichen Meteorologie, Hydrologie, Geomorphologie, Naturgefahren, Ökonomische Aspekte, Recht, Raumordnung und Katastrophenschutz aufgearbeitet, um eine Ursache-Wirkungsanalyse des Ereignisses vorzunehmen, sowie daraus Vorschläge für künftige Verbesserungen („lessons learned“) und entsprechende Umsetzungsstrategien für ein integriertes Hochwassermanagement abzuleiten. Erfolgreiche Umsetzungsstrategien orientieren sich an einem integrierten Hochwassermanagement, dessen Ziel in dem Erreichen eines möglichst geringen Hochwasserrisikos durch Zusammenarbeit aller Betroffenen mit verschiedenen Mitteln in allen Phasen des Risikokreislaufes liegt. Integriertes Hochwassermanagement beinhaltet die vorausschauende, flussgebietsorientierte Entwicklung eines ganzheitlichen Handlungsinstrumentariums sowohl für die Hochwasservorsorge als auch die Bewältigung (FloodRisk I).

Direkt im Anschluss an die Fertigstellung von FloodRisk I kam es 2005 neuerlich zu einem extremen Hochwasser, welches vorrangig den alpinen Teil Österreichs erfasste (3 Todesopfer, über 600 Mio. € Schäden). Da in FloodRisk I einige Themenbereiche nicht behandelt worden waren (Ökologie, Recht, Klimawandel) und neue Problemfelder auftraten (z. B. Massenbewegungen), wurde auf Basis einer unmittelbar nach dem Ereignis durchgeführten Hochwasserdokumentation das Projekt FloodRisk II (Vertiefung und Vernetzung zukunftsweisender Umsetzungsstrategien zum integrierten Hochwassermanagement) begonnen und im Juni 2009 abgeschlossen. In 45 Teilprojekten und von 140 Personen wurden in drei Jahren grundlegende Analysen zu Prozessen und Defiziten im Hochwassermanagement durchgeführt. Das Hauptergebnis besteht in der Darstellung der bisherigen Fortschritte aus FloodRisk I, Empfehlungen und einer vertieften, gesamtheitlichen, konkreten Umsetzungsstrategie zum integrierten Hochwassermanagement.

Die Umsetzung erfordert einerseits gesetzliche Maßnahmen, andererseits Freiwilligkeit in den Bereichen Partizipation, Selbsthilfe im Katastrophenfall und Eigenvorsorge. Dazu bedarf es einer entsprechenden Aufklärung und Kommunikation mit der Bevölkerung. Die Bedeutung der Umsetzung der Ergebnisse aus FloodRisk I und II wurde durch die Hochwässer 2006 an der March und 2009 im Osten und Südosten Österreichs unterstrichen.

Im Rahmen dieses Hefts der Österreichischen Wasser- und Abfallwirtschaft erfolgt eine Darstellung ausgewählter Themen aus FloodRisk II. Die Auswahl bedeutet keine Priorisierung, war aber aufgrund des beschränkten Platzes erforderlich. Weiters ist eine Konzentration auf die wesentlichen Punkte innerhalb der Artikel erforderlich (Details zu FloodRisk I und II finden sich als Downloads, siehe unten).

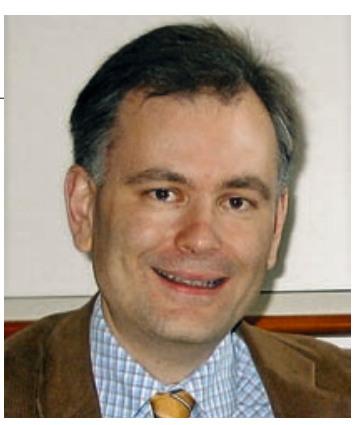

Univ.-Prof. DI Dr. Helmut Habersack 
Das vorliegende Heft besteht aus sechs Artikeln, die nach einem Überblicksbeitrag den Workpackages Hydrologie/Meteorologie, Geomorphologie und Ökologie, Hochwassermanagement, Raumplanung und Recht mit jeweils ausgewählten Themen zugeordnet werden können. Die Workpackages Ökonomie und Katastrophenschutz sind grundsätzlich von gleicher Wichtigkeit und im Einleitungsbeitrag inkludiert.

Abschließend möchte ich mich bei allen Auftraggebern, beim Lenkungskreis (Schimon, Mannsberger, Grill), dem Kernteam (Bürgel, Kanonier, Patek, Pleschko, Prettenthaler, Rudolf-Miklau, Stiefelmeyer), den Workpackage-Leadern (Godina, Hengl, Jungwirth, Prettenthaler, Stiefelmeyer, Patek, Mair, Kanonier), allen ProjektleiterInnen und ProjektbearbeiterInnen für die ausgezeichnete Zusammenarbeit und Unterstützung bei der Durchführung von FloodRisk II bedanken.

Downloads der Hochwasserdokumentationen und Syntheseberichte von FloodRisk I und II finden sich unter:

www.lebensministerium.at

www.umweltbundesamt.at

http://zenar.boku.ac.at

\section{Univ.-Prof. DI Dr. Helmut Habersack}

Universität für Bodenkultur Wien

Department für Wasser, Atmosphäre und Umwelt

Institut für Wasserwirtschaft, Hydrologie und konstruktiven Wasserbau

Muthgasse 107

1190 Wien

helmut.habersack@boku.ac.at 